\title{
Cognitive behavioural therapy improved overall symptomatology in patients with medication resistant psychosis
}

Kuipers E, Garety P, Fowler D, et al. London-East Anglia randomised controlled trial of cognitive-behavioural therapy for psychosis. I: Effects of the treatment phase. BrJ Psychiatry 1997 Oct 1997;171:316-27.

\section{Question}

In patients with medication resistant psychosis, can cognitive behavioural therapy (CBT) improve overall symptomatology?

\section{Design}

Randomised controlled trial with 9 months follow up.

\section{Setting}

3 sites in the UK: Maudsley Trust, Addenbrooke's Hospital Trust, and Norfolk Mental Health Trust.

\section{Patients}

60 patients (mean age $40 \mathrm{y}, 63 \%$ men, mean duration of illness 13 y) with $\geqslant 1$ positive psychotic symptom (eg, delusions or hallucinations) which was distressing, unremitting, and medication resistant (ie, not responsive to $\geqslant 6$ months of an appropriate neuroleptic medication). Patients who had drug, alcohol, or organic problems as primary features were excluded.

\section{Intervention}

28 patients were allocated to CBT plus standard treatment and 32 patients were allocated to standard treatment alone. Standard treatment consisted of case management and medication. CBT was directed at reducing the distressing and disabling effect of psychotic symptoms, improving mood, and engaging the patient actively in working to regulate the risk of relapse and social disability.

\section{Main outcome measures}

Symptoms and functioning measures (Brief Psychiatric Rating Scale [BPRS], Beck Depression Inventory, Beck Hopelessness Scale, and Beck Anxiety Inventory), self concept measures, and clinical outcome.

\section{Main results}

53 patients $(88 \%)$ provided sufficient data to estimate linear trends on the BPRS during the 9 months of therapy. Patients in the CBT group had greater improvement (a 25\% reduction) on the BPRS than those in the control group $(p=0.009)$. The BPRS items which showed the greatest improvement were the 2 items measuring severity of delusional beliefs and the item assessing hallucinations (none reached statistical significance). No differences existed between the treatment groups for all other clinical, symptomatic, or functioning measures. 14 of 28 patients $(50 \%)$ in the CBT group were treatment responders $(\geqslant 5$ point change on the BPRS) compared with 10 of 32 patients (31\%) in the control group $\{\mathrm{p}=0.12\}^{*}$.

\section{Conclusion}

Cognitive behavioural therapy improved overall symptomatology (improvement on the Brief Psychiatric Rating Scale) in patients with medication resistant psychosis.

* p value calculated from data in article.
Source of funding:Department of Health.

For correspondence: Dr E Kuipers, Professor of Clinical Psychology, Institute of Psychiatry, London SE5 8AF, UK. Fax +44 (0)171708 3497

\section{Commentary}

The treatment of schizophrenia consumes about $3 \%$ of total health service spending in the UK. Most of these costs are for the $20-40 \%$ of patients who respond poorly to, or are intolerant of, antipsychotic drug treatment. Over the past 10 years there has been increasing interest, particularly in the UK, in the application of a specific and specifiable psychological technique-CBT-for persistant symptoms in chronic schizophrenia. Anecdotal reports and one randomised controlled trial ${ }^{1}$ support the efficacy of CBT in this patient population.

This study by Kuipers $e t$ al is important. It reports the results of a 3 centre randomised controlled trial of CBT in addition to standard treatment compared with standard treatment alone in patients with treatment resistant psychotic disorders. The experimental treatment package lasted up to 9 months, during which patients were seen initially at weekly intervals, with a median of 15 sessions. Content of the treatment is described in detail.

The experimental group showed greater improvement over 9 months on BPRS total scores. None of the other symptom or social functioning scores showed a difference, however. Improvement was seen particularly for delusions.

The strengths of this study are a strong research team, a well specified intervention, a range of assessments and sophisticated statistical handling of the data. One weakness is the lack of blindness of the outcome assessments, which is difficult but none the less desirable in psychological treatment studies. A second comparison group to control for possible nonspecific effects of increased contact time should also be built in to future studies. Two further multicentre trials of this technique in resistant schizophrenia will shortly report their findings. If these are also positive, we can conclude safely that CBT is effective for some patients with persistent symptoms. The next questions will concern its cost effectiveness and where it stands in comparison with clozapine treatment, also shown to be effective for $30-50 \%$ of patients with refractory symptoms. Head to head trials looking for differential predictors of response to the 2 treatments will be needed to define their positions in future treatment algorithms for chronic schizophrenia.

\section{Shôn Lewis, MD, MRCPsych University of Manchester Manchester, UK}

1 Tarrier N, Beckett R, Harwood S, et al. A trial of two cognitive-behavioural methods of treating drug-resistant residual psychotic symptoms in schizophrenic patients: I. Outcome. $\mathrm{Br} J$ Psychiatry 1993;162:524-32. 